\title{
Effect of Major Axis Length to the Pressure on Ellips
}

\author{
Chairul Imron and Erna Apriliani
}

\begin{abstract}
Fluid concept has been widely applied to solve problems of daily life, one example is the problem of fluid flow around an elliptical cylinder. We have a goal to solve the problem of fluid flow through three cylindrical ellipses using side-by-side configuration. The equation we use to solve the problem is the Navier-Stokes equations, incompressible, viscous and unsteady. We use the finite difference method with a uniform grid and SIMPLE (Semi Implicit Method for Pressure-Linked Equations) algorithms. Results of this study were used to obtain the amount of pressure that is received by an ellipse in the middle and to construct mathematical models. The profile of the fluid flow is simulated by varying the length of the major axis of the ellipse in the middle where $K / 5 a=1.0,1.1,1.2,1.3,1.4$ and 1.5 and Reynolds $R_{e}=3,000$ and the distance between the ellipse is 3 .
\end{abstract}

Index Terms-Three ellips using side-by-side cofiguration, Navier-Stokes equations.

\section{INTRODUCTION}

$\mathbf{T}$ HE car body is designed to reduce drag received by the car, so that the drag is accepted by the body of the car is expected to a minimum, it is expected also when designing the boat trimaran. The basic concept for analyzing drag is also used in various forms ranging from boxes, cylinders, circles, ellipses and other forms of modification. Then we developed with the object of more than one configuration tandem or sideby-side.

Boat which has three hull interrelated with the bridge structure is called the Trimaran. Assuming that the hull shape is an elliptical cylinders, the trimaran is three elliptical cylinder with side-by-side configuration. If the moving particles are assumed as fluid flow, the particles will be moving around the object. The particle movement will be slow or not moving, this is caused by the frictional forces generated by fluid and objects in its path. The particles away from the object will flow faster than the particles close to the object.

Drag will be generated from the flow of fluid through an object. The magnitude of the drag received by the object is influenced by several parameters, one of which is the coefficient of drag. To reduce the drag of an object by adding other, smaller objects are added in front of the main objects called passive control or in addition to the main object. Provision of passive control in front of the main things have

Manuscript received December 8, 2016; accepted December 30 2016. This work is supported by research grant with contract number 31028/IT2.11/PN.08/2016 (23 May 2016), from the research centre of Institut Teknologi Sepuluh Nopember (LPPM ITS), Surabaya, Indonesia.

The authors are with the Department of Mathematics, Institut Teknologi Sepuluh Nopember, Surabaya 60111, Indonesia. Email: \{imron-its, april\}ematematika.its.ac.id been done, the result is a drag on the object that can be reduced up to $48 \%$ compared to without passive controls [1].

The phenomenon of fluid passing an object can be expressed by the concept of the boundary layer, that the shear stress has a very important role in the characteristics of drag around the object [2]. The fluid that flows through a circular cylinder [3], cylinder $D$-types or $I$-types [4], or two cylinders with a tandem configuration [5] will produce a different drag.

The purpose of this paper is to determine the amount of pressure that is received by an elliptical cylinder in the middle as a result of the influence of two elliptical cylinders that flank. In addition, the amount of pressure in the middle of an elliptical cylinder is also influenced by the length of the major axis of the ellipse in the center of the cylinder.

We have discussed the two-dimensional fluid flow in a through two elliptical cylinders configuration with side-byside, with the Reynolds number varies and the distance between an elliptical cylinder is $2 \leq S / a \leq 4$ where $S$ is the distance between the elliptical cylinder and $a$ is the length of the minor axis of the ellipse and the results of this study are at a certain distance is received elliptical cylinder pressure is minimum [6].

Sintu Singha and K.P. Sinhamahapatra conduct research on fluid flow passing through the object of cylindrical single circular with low Reynolds numbers at $45 \leq R_{e} \leq 250$ and perform experiments on the distance between the dividing wall which is $2 \leq H / D \leq 8$ [7]. Sarvghad-Moghaddam Hesam and Nooreddin Navid conducted a study on fluid flow around two circular cylinders arranged on side-by-side with the Reynolds number 100 and 200 for a laminar flow, $10^{4}$ to turbulent flow. Then the position within the cylinder using a ratio that varies at $2 \leq L / D \leq 4$ is $1.5,3,4$ [8].

This paper discusses the flow of fluid passing through three elliptical cylinder configuration with side-by-side to determine the pressure received at four different points with focus on fluid flow and pressure profiles received by the third elliptical cylinder.

\section{NUMERICAL METHOD}

Navier-Stokes equations will be used to complete the fluid that has the following properties: unsteady, viscous and incompressible is

$$
\begin{aligned}
& \frac{\partial \mathbf{u}}{\partial t}+\nabla \cdot \mathbf{u u}=-\nabla P+\frac{1}{R e} \nabla^{2} \mathbf{u} \\
& \nabla \cdot \mathbf{u}=0 .
\end{aligned}
$$

where $R_{e}$ is the Reynolds number, $P$ is the pressure, and $\mathbf{u}$ is the speed. SIMPLE algorithm is used to solve the Navier- 
Stokes equations. The first step taken is to provide an initial value for each variable, and then, get the speed component of the momentum equation by ignoring the earlier pressure components, so that (1) becomes

$$
\frac{\partial \mathbf{u}}{\partial t}=-\nabla \cdot \mathbf{u} \mathbf{u}+\frac{1}{R e} \nabla^{2} \mathbf{u}
$$

right side is solved using finite difference, and the second step is carried out

$$
\frac{\partial \mathbf{u}}{\partial t}=\frac{\mathbf{u}^{* *}-\mathbf{u}^{*}}{\triangle t}=-\nabla P
$$

We now put divergence on both sides, and the result is

$$
\frac{\nabla \mathbf{u}^{* *}-\nabla \mathbf{u}^{*}}{\Delta t}=-\Delta P
$$

by considering (2) then $\nabla \mathbf{u}^{* *}=0$, the equation turns into

$$
\frac{\nabla \cdot \mathbf{u}^{*}}{\Delta t}=-\Delta P
$$

Equation (6) is called Poisson's equation. Completion of the Poisson equation solution will be more quickly to achieve convergence, if using SOR (Successive Over Relaxation)

$$
\left(P_{n}\right)_{i, j}=(1-\omega)\left(\left(P_{n-1}\right)_{i, j}\right)+\omega\left(P_{n}\right)_{i, j}
$$

This equation will yield a value of $P$. The final step is the correction of velocity equation by using the following formula

$$
\frac{\partial \mathbf{u}}{\partial t}=-\nabla P
$$

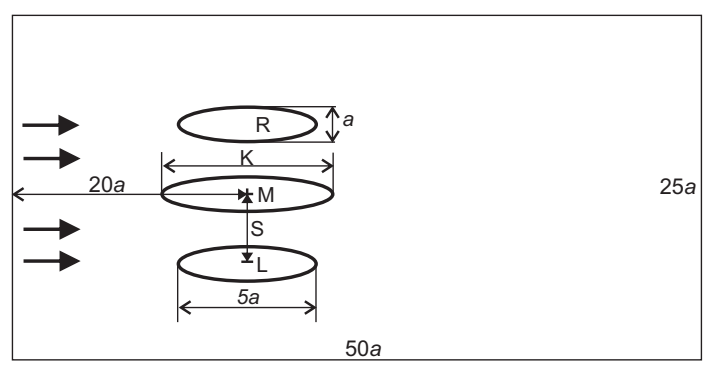

Fig. 1. Schematic of three elliptical cylinders.

\section{MAIN RESULTS}

We will simulate the above problems by establishing a system of measuring $25 a \times 50 a$, where $a$ is the length of the minor axis of an elliptical cylinder. Three eliptical cylinders are top elliptical cylinder $(R)$, bottom elliptical cylinder $(L)$, and an middle elliptical cylinder part $(M)$. An elliptical cylinder $R$ and $L$ has a long axis of the major to the $5 a$ and the long axis of the majors for an elliptical cylinder $M$ varies the $K / 5 a=1.0,1.1,1.2,1.3,1.4$ and 1.5 , and $S$ is the distance between cylinders. The third center of an elliptical cylinder is located on one line and a $20 a$ from the front of the system and Reynolds number is $R_{e}=3000$, as shown in Fig. 1.

A simulation program has a truth value of a good, if the simulation results are closer to the experimental value. Drag coefficient of the experimental results are compared with simulation results which can be seen in Table I.
TABLE I

COMPARISON OF $C_{D}$ OF A CYLINDER CIRCULAR FOR RE $=100$

\begin{tabular}{c|cccc}
\hline Researcher & Imron [9] & Zulh [10] & Lima [11] & Pan [10] \\
\hline$C_{D}$ & 1,358 & 1,4 & 1,39 & 1,32 \\
\hline & & & & \\
\hline & & & & \\
\hline
\end{tabular}

Fig. 2. Pressure point on an elliptical cylinder

The results of the simulation program pressure data received by all of the ellipse. For each elliptical cylinder, we taken four pressure data that the pressure on the front end $(F)$, the rear end $(R r)$, the center of the top side $(T)$ and the center of the bottom side $(B)$ in Fig. 5. The pressure is acceptable for a single elliptical cylinder with sizes varying with Reynolds number $R_{e}=3000$ which can be seen in Table II.

TABLE II

PRESSURE FOR SINGLE ELLIPS WITH $R_{e}=3000$

\begin{tabular}{c|llll}
\hline Size & Front & Rear & Top & Bottom \\
\hline$a \times 5.0 a$ & 0.0001985 & -0.0000469 & -0.0000468 & -0.0000490 \\
$a \times 5.5 a$ & 0.0001987 & -0.0000414 & -0.0000418 & -0.0000441 \\
$a \times 6.0 a$ & 0.0001984 & -0.0000390 & -0.0000388 & -0.0000416 \\
$a \times 6.5 a$ & 0.0001983 & -0.0000376 & -0.0000365 & -0.0000394 \\
$a \times 7.0 a$ & 0.0001984 & -0.0000363 & -0.0000345 & -0.0000375 \\
$a \times 7.5 a$ & 0.0001984 & -0.0000353 & -0.0000326 & -0.0000358 \\
\hline
\end{tabular}

We will discuss the results of the simulation program of three elliptical cylinder with side-by-side configuration with the Reynolds number $R_{e}=3000$ and the distance between the ellipse is $S / a=3.0$, and the ratio of the long axis of the major elliptical cylinder that is central to the axis of the major another elliptical cylinder is $K / 5 a=1.0,1.1,1.2,1.3,1.4$ and 1.5. We take four points from each elliptical cylinder pressure that is on the front end, back end, the upper side and lower side.

Table III represents the data of an elliptical cylinder $M$ a middle elliptical cylinder. Pressure received on the front end $(F)$ of the ellipse has almost the same pressure at different sizes, as well as compared to a single elliptical cylinder, because the point is hardly influenced by the other ellipse. Stresses imposed on the rear end $(R r)$ of the ellipse is almost twice the size compared to the single elliptical cylinder, it is the influence of flow through both slits elliptical cylinder. The pressure point $T$ and $B$ is the pressure on the upper side and lower side of the central ellipse, the pressure received at that point is nearly half of the pressure received by a single ellipse.

TABLE III

PRESSURE OF MIDDLE ELLIPS $M$ With $R_{e}=3000$

\begin{tabular}{c|llll}
\hline Size & Front & Rear & Top & Bottom \\
\hline$a \times 5.0 a$ & 0.0001926 & -0.0000718 & -0.0000267 & -0.0000292 \\
$a \times 5.5 a$ & 0.0001923 & -0.0000706 & -0.0000224 & -0.0000248 \\
$a \times 6.0 a$ & 0.0001923 & -0.0000691 & -0.0000188 & -0.0000212 \\
$a \times 6.5 a$ & 0.0001923 & -0.0000675 & -0.0000156 & -0.0000180 \\
$a \times 7.0 a$ & 0.0001923 & -0.0000656 & -0.0000132 & -0.0000153 \\
$a \times 7.5 a$ & 0.0001922 & -0.0000645 & -0.0000116 & -0.0000133 \\
\hline
\end{tabular}


Table IV is the data of an elliptical cylinder $R$ : a top elliptical cylinder. The size of the first column is the size of the middle elliptical cylinder part, while the size of the top ellipse is the same that $a \times 5 a$. Pressure received on the front end $(F)$ of the ellipse $R$ has a smaller pressure than the single elliptical cylinder, because the point is influenced by the middle ellipse. Stresses imposed on the rear end $(R r)$ of the ellipse has size

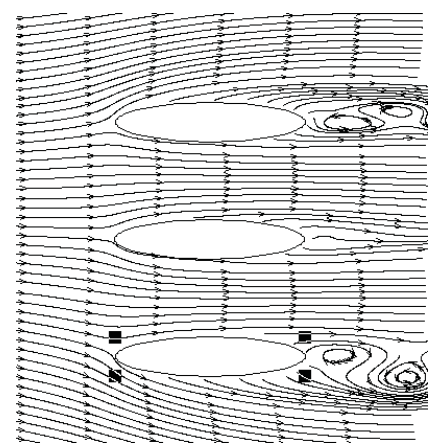

Fig. 3. Flow through the three ellipses with $K=5 a$

of nearly one and a half times compared with single elliptical cylinder, it is the influence of flow through the second elliptical cylinder. The point $R r$ stresses imposed at this point one and a half times larger like Fig. 3 and Fig. 4, it is the influence of the center ellipse. The point $B$ is the pressure on the bottom side of the ellipse above, stresses imposed on the smaller point is nearly half of the stresses imposed by a single ellipse, it is the influence of the second cylinder.

TABLE IV

PRESSURE OF TOP ELLIPS $R$ WITH $R_{e}=3000$

\begin{tabular}{c|llll}
\hline Size & Front & Rear & Top & Bottom \\
\hline$a \times 5.0 a$ & 0.0001767 & -0.0000652 & -0.0000621 & -0.0000241 \\
$a \times 5.5 a$ & 0.0001767 & -0.0000650 & -0.0000620 & -0.0000241 \\
$a \times 6.0 a$ & 0.0001771 & -0.0000644 & -0.0000618 & -0.0000241 \\
$a \times 6.5 a$ & 0.0001776 & -0.0000637 & -0.0000613 & -0.0000237 \\
$a \times 7.0 a$ & 0.0001781 & -0.0000630 & -0.0000608 & -0.0000236 \\
$a \times 7.5 a$ & 0.0001784 & -0.0000637 & -0.0000612 & -0.0000237 \\
\hline
\end{tabular}

Table $\mathrm{V}$ is the data of an elliptical cylinder $L$ : the bottom elliptical cylinder. The size of the first column is the size of the middle elliptical cylinder part, while the size of the bottom elliptical cylinder is the same that $a \times 5 a$. Pressure received on the front end $(F)$ of the ellipse $L$ has a pressure that is similar to the pressure received on the front end of the ellipse $R$ and smaller than the single elliptical cylinder, because the point is influenced by the elliptical $M$, as well as stresses imposed on the rear end point $R r$ of the ellipse $L$ the same as the rear end point $R r$ of the ellipse $R$, i.e. the pressure received is one and a half times that of a single ellipse like Fig. 3 and Fig. 4. Meanwhile, at the point on the ellipse $T$ of elliptic $L$ stresses imposed at this point is one and a half times smaller than a single ellipse, it is the influence of the center ellipse and at the bottom point of the ellipse $B$ of elliptic $L$ is the pressure on the lower side $(B)$ of the ellipse $L$, the pressure received at that point is one and a half larger than the pressure received by the single ellipse this is the influence of both cylinder.

Table III can be used to make the graph in Fig. 5 which is the graph for the pressure at the point the front end $(F)$

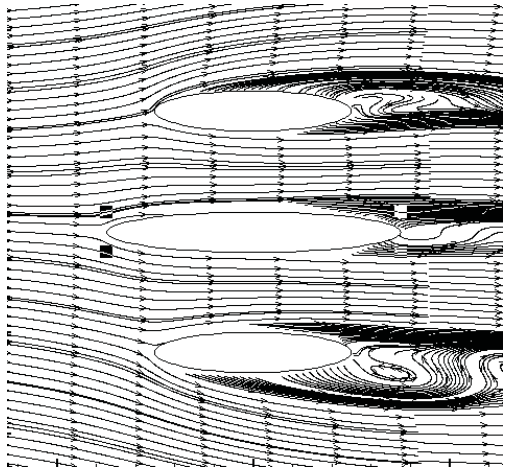

Fig. 4. Flow through the three ellipses with $K=7.5 a$

TABLE V

PRESSURE OF BOTTOM ELLIPS $L$ WITH $R_{e}=3000$

\begin{tabular}{c|llll}
\hline Size & Front & Rear & Top & Bottom \\
\hline$a \times 5.0 a$ & 0.0001607 & -0.0000706 & -0.0000240 & -0.0000704 \\
$a \times 5.5 a$ & 0.0001615 & -0.0000701 & -0.0000244 & -0.0000701 \\
$a \times 6.0 a$ & 0.0001622 & -0.0000698 & -0.0000245 & -0.0000699 \\
$a \times 6.5 a$ & 0.0001635 & -0.0000691 & -0.0000245 & -0.0000693 \\
$a \times 7.0 a$ & 0.0001649 & -0.0000679 & -0.0000244 & -0.0000685 \\
$a \times 7.5 a$ & 0.0001658 & -0.0000670 & -0.0000244 & -0.0000679 \\
\hline
\end{tabular}

and points rear end $R r$ on the middle ellipse $(M)$, it shows that the longer the major axis of the ellipse, then the pressure difference between the front and the end point is very small. The amount of pressure that is received at the point of the front end of the central ellipse can be written in mathematical models, namely

$$
y_{F}=-2 \cdot 10^{-7} x^{3}+3 \cdot 10^{-6} x^{2}-2 \cdot 10^{-5} x+2 \cdot 10^{-4}
$$

and the amount of pressure that is received at the point of the rear end of the central ellipse can be written in mathematical models, namely

$$
y_{R}=-3 \cdot 10^{-7} x^{3}+5 \cdot 10^{-6} x^{2}-3 \cdot 10^{-5} x+2 \cdot 10^{-5}
$$

where $y_{F}$ is the pressure at the front end point, $y_{R}$ is the pressure at the rear end point, and $x$ is the length of the major axis of the center ellipse.

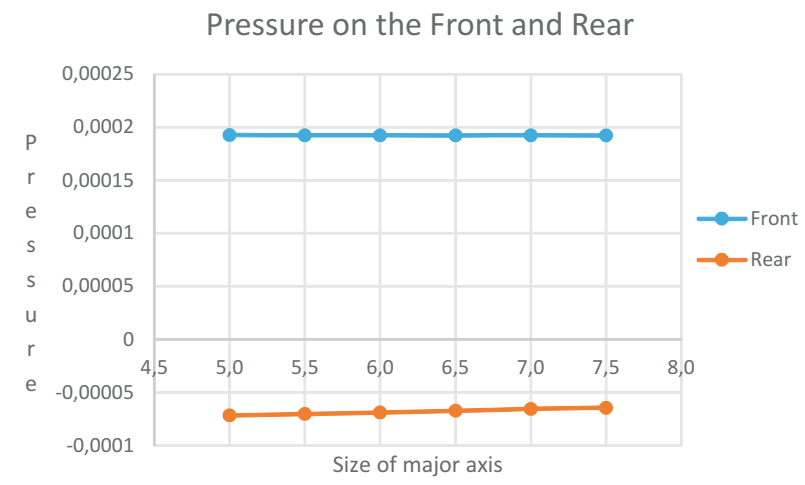

Fig. 5. Pressure on the front and rear of the elliptical middle 


\section{REFERENCES}

[1] F. Bouak and J. Lemay, "Passive control of the aerodynamic forces acting on a circular cylinder," Experimental Thermal and Fluid Science, vol. 16, no. 1-2, pp. 112-121, 1998.

[2] B. Widodo, "The influence of hydrodynamics on pollutant dispersion in the river," Int. J. Contemp. Math. Sciences, vol. 7, no. 45, pp. 2229 $2234,2012$.

[3] A. Ladjedel, B. Yahiaoui, C. Adjlout, and D. Imine, "Experimental and numerical studies of drag reduction on a circular cylinder," World Academy of Science, Engineering and Technology, vol. 77, pp. 357-361, 2011.

[4] T. Y. and W. A. Widodo, "Flow characteristics around a d-type cylinder near a plane wall," in Regional Conferences on Mechanical and Aerospace Technology, 2010.

[5] T. Y. and M. Nuh, "Using of a bluff body cut from a circular cylinder as passive control to reduce aerodynamics forces on a circular cylinder," in The International Conference on Fluid and Thermal Energy Conversion, 2003.

[6] C. Imron and M. Yunus, "Mathematical modeling of pressure on cylindrical ellipse using side-by-side configuration," International Journal of Computing Science and Applied Mathematics, vol. 1, no. 1, pp. 10-11, 2015.

[7] S. Singha and K. Sinhamahapatra, "Flow past a circular cylinder between parallel walls at low Reynolds numbers," Ocean Engineering, vol. 37, no. 8-9, pp. 757-769, 2010.

[8] H. Sarvghad-Moghaddam, N. Nooredin, and B. Ghadiri-Dehkordi, "Numerical simulation of flow over two side-by-side circular cylinders," Journal of Hydrodynamics, Ser. B, vol. 23, no. 6, pp. 792-805, 2011.

[9] C. Imron, B. Widodo, and T. Yuwono, "Numerical simulation of fluid flow around circular and I-shape cylinder in a tandem configuration," Applied Mathematical Sciences, vol. 7, no. 114, pp. 5657-5666, 2013.

[10] D. Z. Noor, M.-J. Chern, and T.-L. Horng, "An immersed boundary method to solve fluid-solid interaction problems," Computational Mechanics, vol. 44, no. 4, pp. 447-453, 2009.

[11] A. L. E. Silva, A. Silveira-Neto, and J. Damasceno, "Numerical simulation of two-dimensional flows over a circular cylinder using the immersed boundary method," Journal of Computational Physics, vol. 189, no. 2, pp. 351-370, 2003. 